\title{
Ultra-stable dye-filled polytetrafluoroethylene thin films
}

\author{
Kostyantyn Grytsenko ${ }^{1 *}$, Sigurd Schrader ${ }^{2}$ and Heiner Detert ${ }^{3}$ \\ ${ }^{1}$ Institute of Semiconductor Physics, pr. Nauki 41, Kyiv, 03650, Ukraine, \\ ${ }^{2}$ University of Applied Sciences Wildau, Bahnhofstrasse, 15745, Germany \\ ${ }^{3}$ Institute of Organic Chemistry, J.Gutenberg University, Duesbergweg10-14, D-55099, Mainz, Germany
}

Received: March 18, 2014; Accepted: May 26, 2014; Published: May 28, 2014

*Corresponding author: Kostyantyn Grytsenko, Institute of Semiconductor Physics, pr. Nauki 41, Kyiv, 03650, Ukraine; email: d.grytsenko@gmail.com

\begin{abstract}
Polytetrafluoroethylene (PTFE) and polyparaphenylene sulphide (PPS) thin films filled with molecules of different dyes were obtained by co-deposition in vacuum. The PTFE matrix prevented aggregation of dye molecules more strongly, than PPS matrix. During heating PTFE matrix kept the dye molecules in not aggregated state up to dye decomposition temperature. Dyes of all types, buried in the PTFE matrix, exhibited high stability toward actions of external factors: light, temperature, acids.
\end{abstract}

Keywords: Dye; PTF; PPS; Nanocomposite; Thin film; Optical spectra; Stability

\section{Introduction}

Recent years revealed breakthrough in the industrial applications of the thin organic functional films. The devices include multi layers with both active and passive layers consisted of the small organic molecules (dyes) and/or polymers. As a rule, the dye solid films are not as stable as necessary for industrial application. To enhance stability of the film optical properties, dye molecules are introduced into polymer matrix by dissolution in solvent and then film is produced by spin coating and other wet techniques. Various sensors made from the organic thin films are described in literature, for example, in the reviews [1,2]. Novel research in this field are concerned not only dye-in-polymer film [3], but three component nanocomposite film as well [4]. In many cases this is a good method to prevent dye molecule aggregation and keep film with necessary optical properties for a long time. Nevertheless, for advanced industrial applications the more stability of the film optical properties is necessary. Diaminonitro-stillbene filled CYTOP films were made by means of laser evaporation in vacuum for the use in electro-optic devices [5]. But with organic compound concentration increase the crystal domains were appeared in the film together with amorphous phase. The matrix material or deposition method gave the film with not good quality. Here we report the one way for the significant improvement of the stability of the dye molecules to action of the environmental factors. Method is based on the simultaneous evaporation and co-deposition on the substrate in vacuum of the polytetrafluoroethylene (PTFE) and dye. This is possible due to utilization of the unique method of the PTFE film deposition in vacuum. PTFE pellets are heated to decomposition temperature in the crucible. Pellets emit volatile gasses, which undergo activation by electron cloud created just above crucible [6-10]. In such deposition method the energetic species able to destroy dye molecules in the gas phase and on the substrate during film growth are absent. PPS and PTFE thin films, filled with dye molecules of various kinds at different concentrations were obtained, their optical properties were studied during light, solvent, temperature actions.

The paper describes behaviour of the dyes in thin film polymer matrices during external actions.

\section{Materials and Methods}

PTFE pellets were used.PPS was presented by Chevron Phillips Chemical Company Ryton $®$ Business Unit. Phthalocyanine (Pc), polymethine and squaraine (SQ) dyes were synthesized in the Institute of Organic Chemistry of National Academy of Sciences of Ukraine, photocromic compounds were given by Photochemistry Center, Moscow, Russian Federation, pyrazine type (St) dyes were presented by Institute of Organic Chemistry of Mainz University, Germany.

Films were deposited using UVN-74 installation equipped with computerised control system, including Pfeiffer pressure meter, optical spectrometer StellarNet and Sigma quartz thickness monitor. Starting pressure in the chamber was $10^{-3} \mathrm{~Pa}$.

Thin PTFE films were deposited in vacuum by the decomposition of the bulk PTFE pellets in the heated crucible and activation of the emitted gasses with cloud of accelerated electrons, localised just at the crucible outlet. The PTFE evaporation scheme is presented at Figure 1.

It was shown that the treatment with electron cloud led to significant changes of the emitted gasses composition. After condensation the secondary PTFE was characterized with mainly linear macromolecular chains and amorphous structure. More details can be found elsewhere [6-10]. Dye was evaporated from thermally heated crucible. The PPS films were deposited by thermal decomposition of PPS powder in the heated crucible. Glass substrates were used. The film thickness was in the 50 - 100 nm range. 


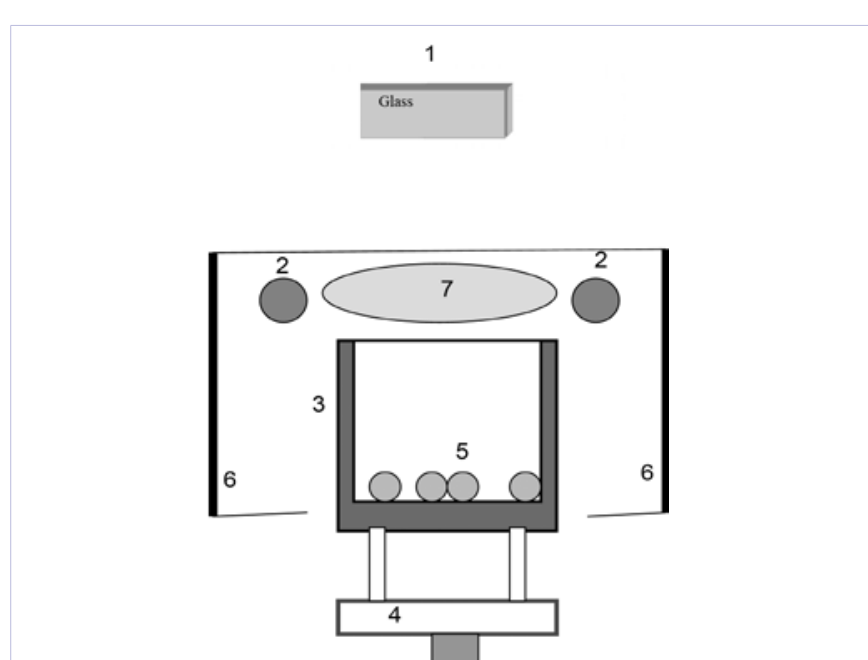

Figure 1: Scheme of the PTFE film deposition:

1 - glass substrate, 2 - hot tungsten wire, 3 - metal crucible, 4 - its holder, 5 - PTFE pellets, 6 - shield, 7 - cloud of electrons.

The measurements of photostationary spectra, kinetics of photocoloration, photobleaching, spontaneous relaxation and photo degradation were carried out using the Ocean Optics spectrometer [11]. The absorption and luminescence spectra of the PTFE and PPS films, filled with acidochromic dye 1 or with squaraine 3 (Figure 2), were measured after immersion of the (A-initial) films in air saturated with dichloromethane (B), followed by methanol (C), followed by acetic acid (D) and finally trifluoroacetic acid (E). All immersions in vapours were about 15 minutes. Polymer films, filled with squaraine 2 were used for studies of the films behaviour during heating. Optical spectrometer Polytec was used for spectra recording in situ during film heating in air in home-made oven.

\section{Results and Discussion}

At first stage of the research the probability to obtain dye- filled PTFE films was shown using well known stable Pc and SQ dyes [12-14]. It was suggested, that dye formed separate phase in the PTFE matrix. The suppression of the dye aggregation and transition to high temperature phase was proportional to dye concentration decrease. Annealing led to a higher order inside the dye nanoparticles (NPs), but not to NPs size increase. The PTFE matrix restricts molecular migration and NPs growth. Variation of the SQ dye side groups led to the different type of aggregation in the SQ-filled PTFE film, which is also dependent on dye concentration $[12,13]$. At small dye concentration and high growth rate, the separate dye molecules were frozen in the PTFE matrix. At higher dye concentration molecules formed the NPs in the PTFE matrix with the structure, equilibrium at the nano-size.

The next research was made testing various dyes with different physical chemical effects used to induce optical changes in the films for possible industrial utilization $[15,16]$.

Photochromic thin films were prepared by co-deposition of PTFE and spirocompounds [11]. The irradiation of the film with ultraviolet light led to transition of initial transparent spiro form to merocyanine form with absorption in visible region. It was found certain differences of photochromic transformation of spiropyrans in solution and in composite films deposited in vacuum. The observed independence of the position and a shape of the absorption band of the photoinduced merocyanine form from concentration of photochromic compounds in PTFE film testified the absence of the well known aggregation for spirocompound molecules. Composite films revealed high stability toward photodegradation and irreversible photochemical transformations. The stability of the photoinduced form is important for preparation of photochromic media for three-dimensional optical memory [11].

The dye filled PTFE film was tested for thermal lithography by focused laser beam with $405 \mathrm{~nm}$ wavelength. The round shaped holes were obtained with their diameter far smaller than diameter of laser beam, due to high melting temperature and<smiles>CN(C)c1ccc(/C=C/c2cnc(/C=C/c3ccc(N(C)C)cc3)cn2)cc1</smiles>

1<smiles>[R]N([R])c1ccc(C2=C([O-])C(=C3C=CC(=[N+]([R])[R])C=C3O)C2=O)c(O)c1</smiles>

2

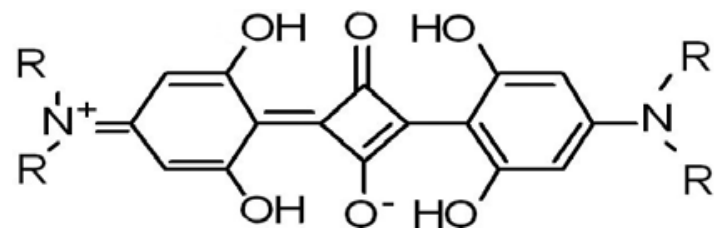

3

Figure 2: Formula of compounds used for co-deposition with polymers:

1 - 2,5-(-4-dimethylaminostyryl)-pyrazine (St); 2 - R= CH3, 2,4-Bis-(4-dimethylamino-2-hydroxyphenyl)cyylobutane-1,3-dione (SQ 2); 3 - R= C4H7, (E,E)-bis[4-(dibutylamino)-2,6-dihydroxyphenyl]squaraine (SQ 3). 

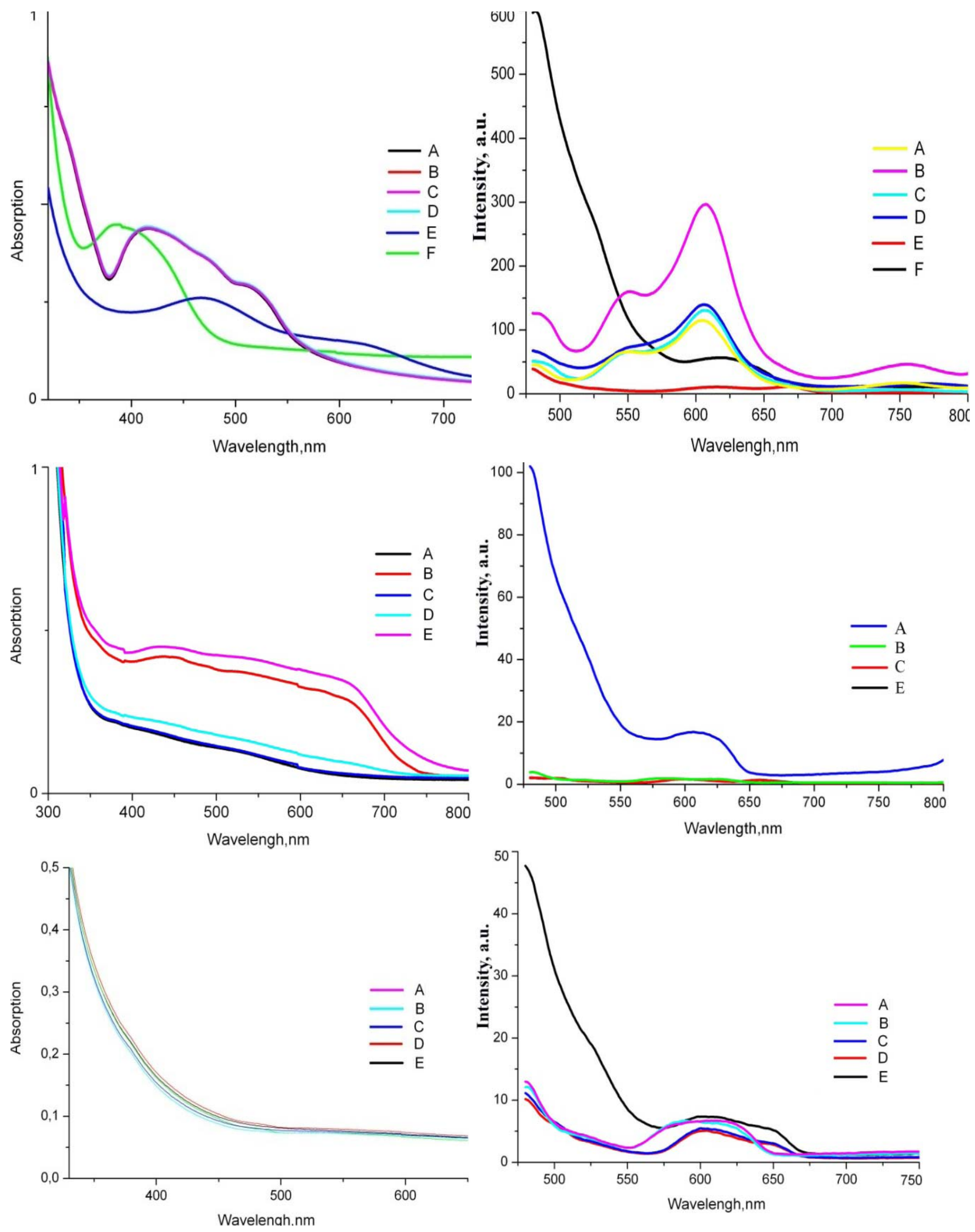

Figure 3: Optical spectra changes of three-component films after vapor treatment:

1 - absorption and 2 - luminescence of the St+PPS film;

3 - absorption and 4 - luminescence of the St+Au+PTFE film;

5 - absorption and 6 - luminescence of the St+As2S3+PTFE film.

Citation: Grytsenko K, Schrader S, Detert H (2014) Ultra-stable dye-filled polytetrafluoroethylene thin films. Nanosci Technol 1(2): 

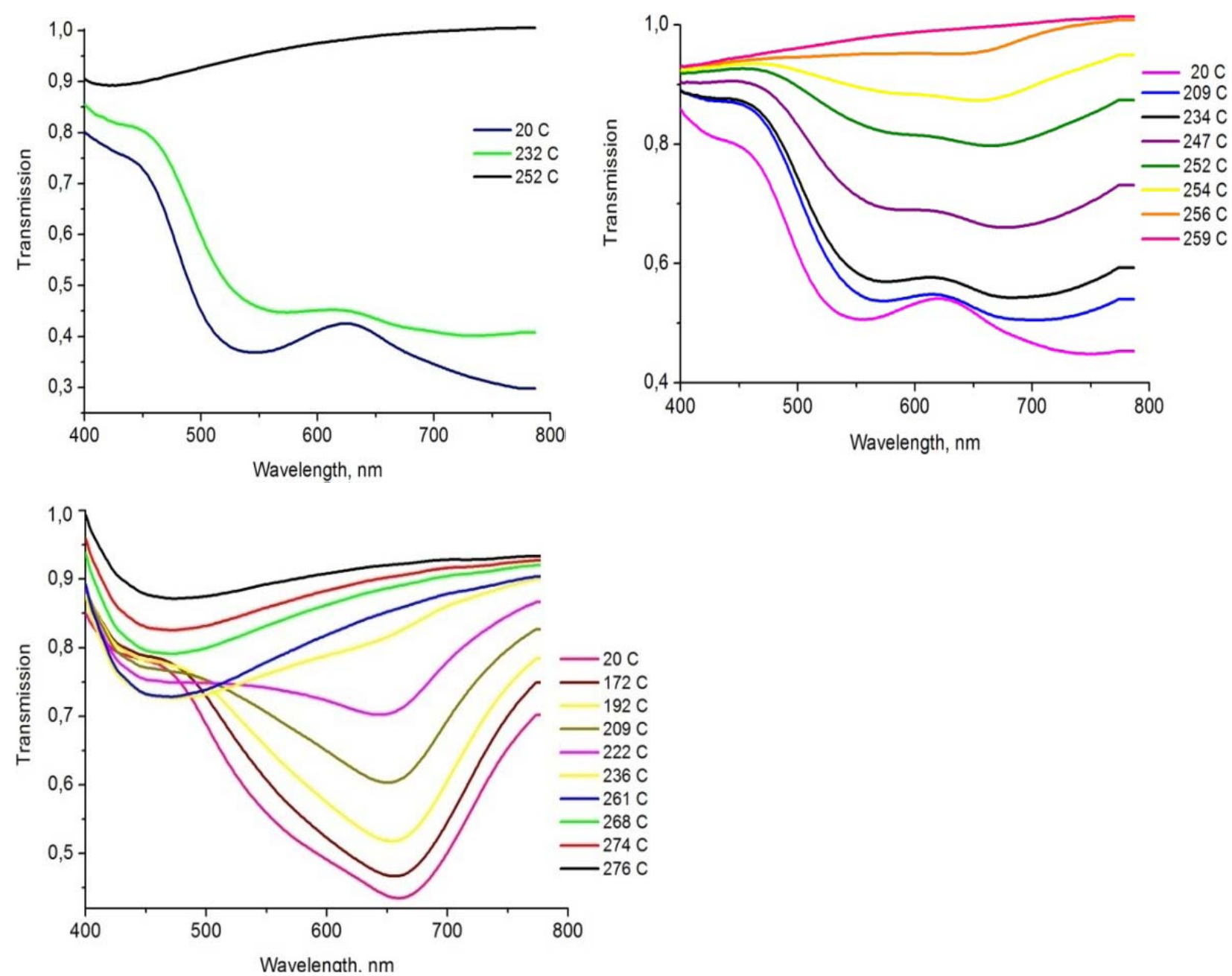

Figure 4: Evolution of the optical spectra of SQ-filled polymer films during heating: 1 - pure SQ film; 2 - SQ in PPS; 3 - SQ in PTFE.

high viscosity of the PTFE melt. The film is useful for super high density optical memory [15].

Two component systems of all type of dyes in the PTFE showed no any absorption or luminescence changes in all vapors (B-E) even at high dye load. But the same dyes showed optical changes while in another polymer, for example, SQ 3 [16]. At low dye load the absence of the optical change can be explained by steric factor, but at high (30\%) concentration of the dye in the film the result seems very strange. The same all dyes but in PPS matrix revealed changes in absorption and luminescence spectra under the same conditions. The next experiments were made using PTFE matrix filled with both dye and inorganic compounds. In comparison with dye-in-PTFE systems, three component films with inorganic and dye nanoparticles (NPs) in PTFE matrix are sensitive toward vapors. Figure 3 presents the absorption and luminescence spectra of the films after vapors action. $\mathrm{St}+\mathrm{Au}+\mathrm{PTFE}$ film revealed changes in absorption spectrum, luminescence was suppressed. $\mathrm{St}+\mathrm{As}_{2} \mathrm{~S}_{3}+\mathrm{PTFE}$ film revealed no absorption change, but detectable luminescence change. These results show, that three component organic-inorganic thin film with PTFE matrix can be used for aggressive vapor optical detection and recognition.

The experiments showed, that the PTFE even in thin film form has strong ability to stabilize the dyes incorporated in it toward all environmental factors. It is hardly possible to explain the results as the sum of the dye and PTFE properties in the thin nanocomposite film. The diffusion of vapors and gases should take a few second through film with thickness of several tens of nanometers. The inorganic NPs can absorb gaseous molecules by such a way supply them into PTFE film toward dye NPs. The PTFE macromolecules deposited by developed method contained electric charge [17]. Perhaps this charge does not allow molecules of the gasses penetrate into film bulk. But what the mechanism of suppression of phototransformations of the spirocompound molecules in charged PTFE environment is not clear. The discovered experimental results deserve farther studies for both scientific understanding and for industrial applications.

The thermal stability of the different polymethine dyes in PTFE matrix was studied in comparison with the properties of 
the same dyes in the PPS matrix. Figure4 presents evolution of the spectra of the SQ 2 -filled polymer films with dye concentration in the range 10-15 vol. \% during heating.

The effect of the polymer matrix on the film optical properties changes during heating is clearly seen. It should be mentioned, that SQ optical band splitting or widening in as-deposited PPS matrix are stronger than in as-deposited PTFE matrix. The SQ molecules aggregation in PPS matrix decomposed at the temperature close to dye decomposition itself [18]. At the temperature at which SQ-filled PPS films became transparent, dye-filled PTFE films still revealed some optical absorption. The PTFE matrix kept the SQ molecules in the not aggregated state up to SQ decomposition temperature. The PTFE film filled with SQ exhibited change of $650 \mathrm{~nm}$ absorption band into 450 $\mathrm{nm}$ band. Film was not completely transparent up to $280^{\circ} \mathrm{C}$. The effect can be explained by SQ molecule decomposition, while the decomposition products were kept in the PTFE matrix.

\section{Conclusions}

New nanocomposite thin film material was produced by codeposition of PTFE and dye in vacuum. The films revealed unique stability of the optical properties of the buried dye nanoparticles under almost all environmental actions. The nanocomposite films are perspective for various photonic applications.

\section{Acknowledgments}

Thanks for the financial support with STCU 5709 Grant and Project 5709-2 funded by National Academy of Sciences of Ukraine. Thanks to DAAD for supply of the Polytec spectrometer

\section{References}

1. Basabe-Desmonts L, Reinhoudt DN, Crego-Calama M (2007) Design of fluorescent materials for chemical sensing. Chem Soc Rev 36(6): 993-1017.

2. Korostynska O, Arshak K, Gill E, Arshak A (2007) Review on State-of-theart in Polymer Based pH Sensors. Sensors 7: 3027-3042 .

3. Mathew RJ, Raj S, Dhesingh RS (2013) PMMA-based Stimuli-responsive Flexible Thin-films. Chemistry Letters 42(11): 1360-1362.

4. Sarkisov SS, Czarick M, Fairchild BD, Liang Y, Kukhtareva T et al. (2013) Colorimetric polymer-metal nanocomposite sensor of ammonia for the agricultural industry of confined animal feeding operations. Opt Eng 53(2): 021107.

5. Kannari F, Fujii T, Shima H, Matsumoto N (1996) Electrical and optical characteristics of organic thin films fabricated by laser ablation. Appl Surf Sci 96-98: 625-629.
6. GritsenkKP, KhimchenkoYu I,Lantoukh GV(1987) Deposition ofbarrier sublayer of optical carrier by evaporation of polytetrafluoroethylene in vacuum with ionisation of degradation products. Optical recording information, Naukova Dumka, Kyiv, Ukraine. 85-88.

7. Gritsenko K (1991) Mechanism of PTFE film growth in vacuum. Ukrainian Chem J 57(7): 782-784.

8. Gritsenko KP Krasovsky AM (2003) Thin film deposition of polymers by vacuum degradation. Chem Rev 103( 9): 3607-3649.

9. Gritsenko KP, Capobianchi A, Convertino A, Friedrich J, Schulze RD et al. (2005)Polymer-metal composite thin film prepared by co-evaporation in vacuum. In (Eds.) Iwamori S Polymer surface modification and polymer coatings by dry process technologies, Research Signpost, Kerala. Pp: 85109.

10. Grytsenko KP (2008) Growth mechanism, properties and applications of vacuum-deposited PTFE films. Russian J. Chem. Soc L II (3): 112123.

11. Grytsenko KP, Machulin VF, Ait AO, Gorelik AM, Kobeleva OI et al. (2010) Photochromic films prepared by vacuum co-deposition of polymer and spiropyrans. Optical Memory and Neural Networks 19(3): 254-259.

12. Gritsenko KP (1995) Laser radiation action on phthalocyanine-filled PTFE film. Thes. Conference "Polymer composites-95", Soligorsk 6566.

13. Gritsenko KP, Dimitriev OP, Kislyk VV, Getsko OM, Schrader S et al. (2002) Dye-fluoropolymer nanocomposite film deposition in vacuum". Colloids \& Surf A Physchem Eng Aspects 198-200: 625-632.

14. Gritsenko KP, Dimitriev OP, Grinko DO, Schrader S, Brehmer L (2003) Dye-filled polymer film formation in a vacuum. Proc SPIE 4833: 487-492.

15. Grytsenko, K., Lytvyn, P., Navozenko, O., Kolomzarov, Yu., Briks, J., Kurdukov, V., Slominskii, Yu., Tolmachev, O., Ksianzou, V., Schrader, S. "Evaporable dyes with absorption in blue region", Conference Nanotechnology and nanomaterials, August 29 -- September 1, 2013, Bukovel, Abstr. Book: p.183.

16. Balbo Block, M. A. and Hecht S. (2004). Alternating (Squaraine-Receptor) Sensory Polymers: Modular One-Pot Synthesis and Signal Transduction via Conformationally Controlled Exciton Interaction, Macromolecules, 37: 4761-4769.

17. Grytsenko K, Kolomzarov Yu, Lytvyn P, Beyer H, Ksenzou V, et al. (2011) Effect of magnetic field on film formation by means of polytetrafluoroethylene decomposition in vacuum. Materials Technolog Tools 16(4): 56-59.

18. Grytsenko K, Navozenko O, Kolomzarov Yu, Kryuchin A, Tolmachev O ey al. (2012) Optical properties of dye-filled polymer films, deposited in vacuum. Data Record Storage \& Proc 14(1): 3-9. 